\title{
Flavonoid diosmetin increases ATP levels in kidney cells and relieves ATP depleting effect of ochratoxin A
}

\author{
Miklós Poór ${ }^{\mathrm{a}}$, Balázs Veres ${ }^{\mathrm{b}}$, Péter B. Jakus ${ }^{\mathrm{b}}$, Csenge Antus ${ }^{\mathrm{b}}$, Gergely Montskóa ${ }^{\mathrm{a}}$ Zita Zrínyi ${ }^{\mathrm{a}}$, \\ Sanda Vladimir-Kneževićc ${ }^{c}$, József Petrik ${ }^{\mathrm{d}}$, Tamás Kőszegi ${ }^{\mathrm{a}, *}$ \\ a Institute of Laboratory Medicine, University of Pécs, Pécs H-7624, Hungary \\ ${ }^{\mathrm{b}}$ Department of Biochemistry and Medical Chemistry, University of Pécs, Pécs H-7624, Hungary \\ ${ }^{c}$ Department of Pharmacognosy, Faculty of Pharmacy and Biochemistry, University of Zagreb, HR-10000 Zagreb, Croatia \\ ${ }^{\mathrm{d}}$ Department of Medical Biochemistry and Haematology, Faculty of Pharmacy and Biochemistry, University of Zagreb, HR-10000 Zagreb, Croatia
}

\section{A R T I C L E I N F O}

\section{Article history:}

Received 15 November 2013

Received in revised form 17 January 2014

Accepted 23 January 2014

Available online 31 January 2014

\section{Keywords:}

Diosmetin

ATP

Flavonoids

Ochratoxin A

Kidney cells

\begin{abstract}
A B S T R A C T
Diosmetin (DIOS) is a flavone aglycone commonly occurring in citrus species and olive leaves, in addition it is one of the active ingredients of some medications. Based on both in vitro and in vivo studies several beneficial effects are attributed to DIOS but the biochemical background of its action seems to be complex and it has not been completely explored yet. Previous investigations suggest that most of the flavonoid aglycones have negative effect on ATP synthesis in a dose dependent manner. In our study 17 flavonoids were tested and interestingly DIOS caused a significant elevation of intracellular ATP levels after 6- and 12-h incubation in MDCK kidney cells. In order to understand the mechanism of action, intracellular ATP and protein levels, ATP/ADP ratio, cell viability and ROS levels were determined after DIOS treatment. In addition, impacts of different enzyme inhibitors and effect of DIOS on isolated rat liver mitochondria were also tested. Finally, the influence of DIOS on the ATP depleting effect of the mycotoxin, ochratoxin A was also investigated. Our major conclusions are the followings: DIOS increases intracellular ATP levels both in kidney and in liver cells. Inhibition of glycolysis or citric acid cycle does not decrease the observed effect. DIOS-induced elevation of ATP levels is completely abolished by the inhibition of ATP synthase. DIOS is able to completely reverse the ATP-depleting effect of the mycotoxin, ochratoxin A. Most probably the DIOS-induced impact on ATP system does not originate from the antioxidant property of DIOS. Based on our findings DIOS may be promising agent to positively influence ATP depletion caused by some metabolic poisons.
\end{abstract}

(c) 2014 Elsevier B.V. All rights reserved.

\section{Introduction}

Flavonoids are natural polyphenols with well-known antioxidant and scavenger characteristics [1]. Furthermore, flavonoids show cytoprotective effects and are considered to be promising agents in prevention and/or therapy of numerous disorders [1-4]. Previous studies highlighted that besides their scavenger property flavonoids exerted influence on the activity of several enzymes, transporters and on cell signaling pathways [1]. Interestingly, based on our current knowledge all of the previous investigations suggested that at least some of the studied flavonoids were able to decrease intracellular ATP levels [5-9]. Chinnam et al. described the inhibition of Escherichia coli (E. coli) ATP synthase on purified $\mathrm{F}_{1}$

* Corresponding author. Address: Institute of Laboratory Medicine, University of Pécs, H-7624 Pécs, Ifjúság u. 13, Hungary. Tel.: +36 72536 120; fax: +36 72536121.

E-mail address: koszegit@freemail.hu (T. Kőszegi). subunit or membrane bound $\mathrm{F}_{1}$ Fo complexes related to different dietary bioflavonoids [5]. Li et al. observed similar inhibitory effect of theaflavins on E. coli ATP synthase [6]: They suggested the $\mathrm{F}_{1}$ part as the primary site of inhibition and they also reported the inhibition of electron transport chain. In another study, Kanno et al. found naringenin-induced significant decrease of intracellular ATP levels and increased dose-dependent hyperpolarization of mitochondrial membrane potential in HL-60 cells [7]. Moreover, Mathiesen et al. and Dorta et al. reported uncoupled oxidative phosphorylation due to C-methylated flavonoids [8] and substantial respiratory chain inhibition by quercetin and galangin [9] in rat liver mitochondria. In our literature survey we have not found any references observing that certain flavonoids might increase intracellular ATP levels.

Diosmetin (DIOS; Fig. 1) is a flavone aglycone occurring mainly in citrus species (and also in related products e.g. lemon or bergamot juices) and in olive (Olea europaea L.) leaves [10-14]. 

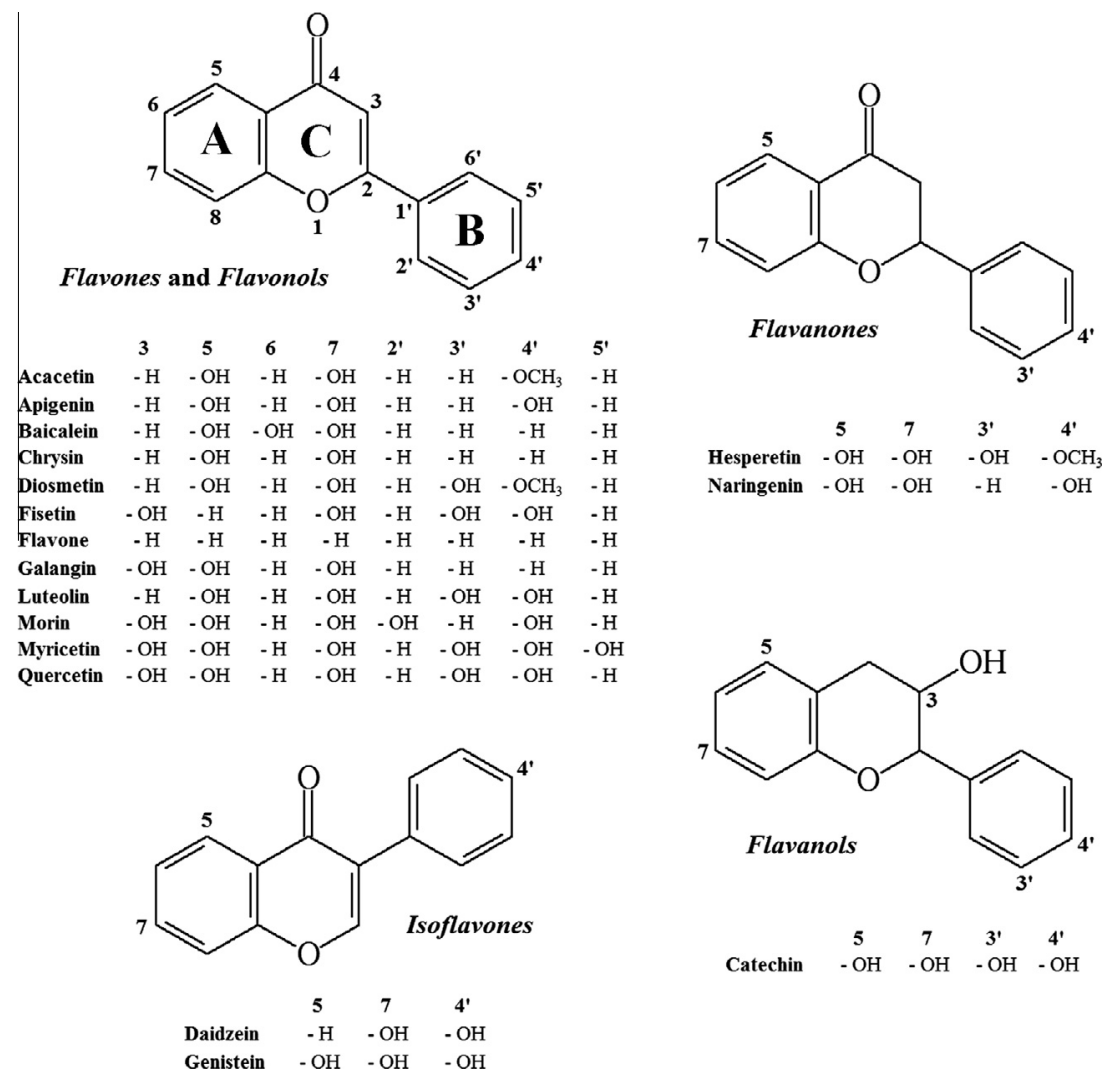

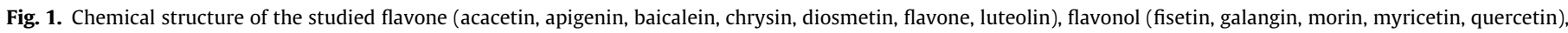
flavanone (hesperetin, naringenin), flavanol (catechin) and isoflavone (daidzein, genistein) aglycones.

Furthermore, it is widely known that the glycoside of diosmetin called diosmin is the active ingredient of various vasoprotective medications, for example Detralex ${ }^{\odot}$ or Daflon $^{\odot}$ (containing 450 mg diosmin) $[15,16]$, which are capillary stabilizing agents. In addition, there have been described several other positive effects attributed to diosmin in recently published studies both in vitro and in vivo, indicating a complex mechanism of action [17-22]. Previous pharmacokinetic investigations showed that only the aglycone DIOS can enter the bloodstream (no diosmin was detected in patent's blood plasma) $[23,24]$. After administration of $450 \mathrm{mg}$ oral dose of diosmin, about $1.0-1.5 \mu \mathrm{M}$ peak concentrations of DIOS were reported in the plasma (studied in healthy human volunteers) [24]. The above listed facts support that additional investigations are needed to explore further potential biochemical effects of DIOS.

The mycotoxin, ochratoxin A (OTA) is a widely spread food contaminant $[25,26]$. Principally nephrotoxic and carcinogenic effects are attributed to OTA but several other adverse effects are also assumed [27]. Previous studies suggested a multiple mechanism in the mode of action of OTA; however one of the most pronounced adverse impacts seems to be its very strong ATP depleting effect [27]. To our current knowledge there has not been reported any substance to be suitable to alleviate the negative effect of OTA on ATP synthesis.

In our present study 17 different flavonoid aglycones were examined from the flavone, flavonol, flavanone, flavanol and izoflavone molecular groups (Fig. 1) to explore their influence on intracellular ATP levels in MDCK kidney-derived cell line using primarily the luciferin-luciferase ATP dependent bioluminescent reaction. In agreement with the previous studies most of the applied flavonoids caused no or only a mild decrease of intracellular ATP concentrations [5-9]. In contrast, the flavone DIOS exerted a significant increase in ATP levels therefore, our experiments were focused on the investigation of this aglycone. In order to confirm our observation ATP/ADP ratios were also determined using HPLC-UV technique. Furthermore, different enzyme inhibitors were applied to get more information on the observed impact of DIOS; and the effect of DIOS on mitochondrial oxygen consumption was also tested using rat liver mitochondria. Finally, combined treatment of MDCK cells with OTA and DIOS was studied. Our results showed a considerable elevation of intracellular ATP levels after short (6-12 h) incubations with micromolar DIOS concentrations $(5-15 \mu \mathrm{M})$ that fell to the control level after $24 \mathrm{~h}$. Using the above described techniques we could not determine the exact mechanism of action of DIOS. Because of the very diverse biochemical effects of DIOS further extensive investigations should be performed. On the other hand, we could clearly show that DIOS was suitable to offset OTA-mediated ATP depletion and was even able to completely restore ATP levels in MDCK cells.

\section{Materials and methods}

\subsection{Chemicals}

All chemicals and reagents were of analytical grade. Diosmetin (DIOS), acacetin, hesperetin (from Extrasynthese), apigenin, chrysin, myricetin, quercetin (from Fluka), daidzein, luteolin (from Indofine), baicalein, catechin, fisetin, flavone, galangin, genistein, morin, naringenin, ochratoxin A (OTA), Dulbecco's Modified Eagle's Medium (DMEM) - high glucose $(4500 \mathrm{mg} / \mathrm{L})$, fetal bovine serum (FBS), oligomycin A (OmA), 3-bromopyruvic acid (3-BP), bovine serum albumin (BSA), adenosine 5 '-triphosphate (ATP), adenosine diphosphate (ADP), penicillin/streptomicyn solution, trypsin solution, sodium dodecyl sulfate (SDS), Triton X 100 (all from Sig- 
ma-Aldrich), Bioluminescent ATP Assay Kit CLSII (from Roche), phenylmethylsulfonyl fluoride (PMSF) and fluorescamine (from Serva), calcein aceoxymethyl ester (CAM) and 6-carboxy-2',7'dichlorodihydrofluorescein diacetate (C400; from Invitrogen) were used as received. $2000 \mu \mathrm{M}$ flavonoid and $5000 \mu \mathrm{M}$ OTA stock solutions were prepared in 96\% ethanol (from Reanal, spectroscopic grade) and kept at $4{ }^{\circ} \mathrm{C}$ protected from light.

\subsection{Instrumentation}

Hitachi F-4500 fluorescence spectrophotometer and Berthold Lumat LB9507 luminometer were used for fluorescent and luminescent experimentations. Measurements were done in the presence of air at room temperature. Promega GloMax ${ }^{\circledR}-$ Multi Microplate Multimode Reader was used for ROS-measurements.

Chromatographic analysis of ATP/ADP concentrations was performed on a Dionex Ultimate 3000 (Dionex Corp., Sunnyvale, CA, USA) analytical HPLC system composed of a binary gradient pump (HPG-3200SD), an autosampler (WPS-3000RS), a thermostatted column compartment (TCC-3000RS) and a UV/VIS multiple wavelength detector (MWD-3000RS).

\subsection{Tissue cultures}

MDCK (cocker spaniel, kidney, immortalized, ATCC: CCL 34) and HepG2 (human, liver, hepatocellular carcinoma, ATCC: HB-8065) adhering cells were cultured in DMEM (high glucose) with $10 \%$ FBS plus penicillin $(100 \mathrm{U} / \mathrm{ml})$ and streptomycin $(100 \mu \mathrm{g} / \mathrm{ml})$ in $25 \mathrm{~cm}^{2}$ sterile plastic flasks (VWR) and in 96-well sterile plastic plates (VWR). Cells were kept at $37{ }^{\circ} \mathrm{C}$ in the presence of $5 \% \mathrm{CO}_{2}$ in humidified atmosphere. Cells were cultured for 2 days in flasks then trypsinized and resuspended. $2 * 10^{4}$ MDCK cells and $1 * 10^{4}$ HepG2 cells per well were dished out to 96-well plates (150 $\mu \mathrm{L}$ medium/well, counted with a Bürker-type chamber). After $16 \mathrm{~h}$ the medium was replaced and the incubation was started using 5-15 $\mu \mathrm{M}$ flavonoid concentrations. Samples were obtained and analyzed after 6,12 and $24 \mathrm{~h}$ incubations. All sterile work was carried out in an Aireguard-126300 (Nuaire) vertical laminar box.

\subsection{Bioluminescent ATP determination}

Intracellular ATP concentrations were quantified using luciferin-luciferase bioluminescent reaction. Sample preparation, measurements and data analysis were done using the previously published method [28] without modifications applying internal standardization. After subjecting cells to the appropriate treatments, cells were washed twice with cold PBS $(150 \mu \mathrm{L})$ thereafter $150 \mu \mathrm{L}$ lysis buffer $\left(0.2 \mathrm{M}\right.$ borate buffer, $10 \mathrm{mM} \mathrm{MgSO}_{4}, 0.1 \%$ Triton $\mathrm{X} 100, \mathrm{pH} 9.2$ ) was added to the wells and the plates were kept at room temperature for $15 \mathrm{~min}$. $50 \mu \mathrm{L}$ cell lysates were pipetted into $75 * 12 \mathrm{~mm}$ disposable plastic cuvettes (Sarstedt) containing $100 \mu \mathrm{L}$ luciferin-luciferase reagent and $850 \mu \mathrm{L}$ ATP measuring buffer (0.1 M TRIS-acetate buffer, $10 \mathrm{mM} \mathrm{MgSO}_{4}, 2 \mathrm{mM} \mathrm{Na}_{2}$ EDTA, $0.1 \%$ Triton X 100, pH 7.75).

\subsection{Determination of ATP/ADP ratio by HPLC-UV}

To further verify our results a distinct ATP extraction method and instrumentation were also applied. First the medium was removed and cells were washed twice with $150 \mu \mathrm{L}$ ice-cold PBS then $100 \mu \mathrm{L} 10 \%$ cold $\left(4^{\circ} \mathrm{C}\right)$ perchloric acid solution was added to the system. After incubation ( $5 \mathrm{~min}$. on ice) every $80 \mu \mathrm{L}$ extract was neutralized with $24 \mu \mathrm{L} 20 \% \mathrm{KOH}$ solution (with $1.5 \mathrm{M}$ $\mathrm{K}_{2} \mathrm{HPO}_{4}$ ) and incubated for $2 \mathrm{~min}$. on ice. Then samples were centrifuged $\left(4^{\circ} \mathrm{C}, 10000 \mathrm{RPM}, 5 \mathrm{~min}\right.$ ) to get rid of the resulting potassium perchlorate precipitate. $40 \mu \mathrm{L}$ from the supernatant was injected to the instrument. The separation of ADP and ATP was carried out according to the method described by Kochanowski et al. [29] with some modifications. Buffer "A" was prepared by adding $8 \mathrm{mM}$ of tetrabutylammonium bisulfate (Sigma-Aldrich) to $100 \mathrm{mM}$ potassium phosphate (Spectrum 3D) buffer ( $\mathrm{pH} 6.5$ ), while buffer "B" was constructed using 70\% buffer "A" and 30\% methanol (Molar Chemicals). Both buffers were filtered on $0.2 \mu \mathrm{m}$ filters (Merck) prior to use. Separation was carried out at $40{ }^{\circ} \mathrm{C}$ on a Supelco Discovery C18 column $(150 \times 4.6 \mathrm{~mm}, 5 \mu \mathrm{m}$ particle size; Sigma-Aldrich) with a flow rate of $1 \mathrm{ml} / \mathrm{min}$. The gradient profile was shortened compared to the method of Kochanowski et al. [29] according to the following description: from 0 to $5 \mathrm{~min} 100 \%$ " $A$ ", then from 5 to $20 \mathrm{~min}$ a linear raise of "B" to $77 \%$, and from 20.5 to 28 min $100 \%$ "A" again to achieve column re-equilibration. All chromatograms were analyzed at $254 \mathrm{~nm}$.

\subsection{Determination of total protein concentrations with fluorescamine reagent}

Our previously published method was used for total protein measurements without modifications [28]. Both ATP detection (lysis with Triton-borate buffer) and intracellular protein determination were performed from the same well. To prevent proteolysis the lysis buffer was completed with $0.2 \mathrm{mM}$ PMSF. $75 \mu \mathrm{L}$ lysates were added to $2000 \mu \mathrm{L}$ borate buffer $(0.2 \mathrm{M}$, pH 9.2) thereafter $50 \mu \mathrm{L}$ fluorescamine solutions ( $10 \mathrm{mM}$; in DMSO) were vortexed into the samples. Fluorescence emission intensities were determined at $475 \mathrm{~nm}\left(\lambda_{\text {exc }}=394 \mathrm{~nm}\right)$, then BSA-standardized total protein concentrations were calculated.

\subsection{Calcein viability assay}

Calcein acetoxymethyl ester (CAM) viability assays were performed based on our previously published method without modifications [28]. After two washing steps with $150 \mu \mathrm{L}$ PBS cells were treated with $150 \mu \mathrm{L}$ CAM solution/well $(1.6 \mu \mathrm{M}$ CAM in PBS $)$ and incubated for $60 \mathrm{~min}$ at $37{ }^{\circ} \mathrm{C}$ in the dark. Then the medium was removed and cells were washed twice with PBS. Thereafter $150 \mu \mathrm{L}$ lysis buffer (0.1\% SDS in PBS) was added to each well and incubated for $10 \mathrm{~min}$ at room temperature. $100 \mu \mathrm{L}$ cell lysates were added to $2000 \mu \mathrm{L}$ PBS then fluorescence emission intensities were determined $\left(\lambda_{\mathrm{exc}}=480 \mathrm{~nm}, \lambda_{\mathrm{em}}=515 \mathrm{~nm}\right)$.

\subsection{Investigation with enzyme inhibitors}

In order to obtain more information on the mechanism of action of DIOS, enzyme inhibitors were applied: sodium fluoride $(\mathrm{NaF})$ is a known inhibitor of enolase enzyme therefore it obstructs glycolysis, 3-bromopyruvic acid (3-BP) inhibits both hexokinase and pyruvate dehydrogenase enzymes [30,31], finally oligomycin A (OmA) inhibits the $F_{O}$ part of ATP synthase. Cells were treated with $10 \mu \mathrm{M}$ DIOS in the absence and in the presence of different enzyme inhibitors for $12 \mathrm{~h}$ using the previously described conditions (Section 2.3.).

2.9. Isolation of rat liver mitochondria and determination of its oxygen consumption

Wistar rats were purchased from Charles River Hungary Breeding Ltd. The animals were kept under standardized conditions; tap water and rat chow were provided ad libitum. Animals were treated in compliance with approved institutional animal care guidelines. Rat liver mitochondria were prepared by the previously described standard protocol [32]. Liver was squeezed through a liver press. All isolated mitochondria were purified by Percoll 
gradient centrifugation [33]. Mitochondrial oxygen consumption was assessed by a Clark type oxygen microelectrode. The mitochondrial respiration was measured in the presence of different concentrations of DIOS applying the previously published protocol [34]. Results were assessed from the original registration curve.

\subsection{Determination of intracellular ROS levels}

Intracellular ROS levels were determined using the oxidationsensitive 6-carboxy-2', $7^{\prime}$-dichlorodihydrofluorescein diacetate (C400) fluorescent dye. C400 is an acetylated fluorescein derivative; the non-fluorescent molecule is converted to fluorescent form by intracellular esterases and oxidation by ROS which occurs inside the cells $[35,36]$. After subjecting cells to the appropriate treatment, cells were treated with $4 \mu \mathrm{M} \mathrm{C} 400$ for $2 \mathrm{~h}$ at $37^{\circ} \mathrm{C}$. Fluorescence measurement was performed on a Promega GloMax $^{\circledR}$-Multi Microplate Multimode Reader, using 490/510570 excitation/emission wavelengths.

\subsection{Statistics}

All experiments were repeated at least three times. Values are presented as means \pm SD. Statistical analyses were performed with One-Way ANOVA using IBM SPSS Statistics software (Version 20). $P<0.05$ was set as the level of significance.

\section{Results and discussion}

\subsection{Effects of flavonoid aglycones on ATP levels of MDCK cells}

First the effects of 17 different flavonoid aglycones were examined on intracellular ATP levels in MDCK cells applying $10 \mu \mathrm{M}$ aglycone concentration and 12-h incubation time. Fig. 2a shows that among the tested flavonoids only DIOS caused significant elevation of ATP concentrations after 12-h treatment. Furthermore, minor (non-significant) increase of ATP was caused by apigenin. As Fig. 1 demonstrates there is some structural homology between DIOS and apigenin: both derivatives belong to the flavone molecular group (presence of 2,3-double bound and absence of 3-hydroxyl group) and possess the 5- and 7-hydroxyl groups. On the other hand, apigenin does not present the $3^{\prime}$-hydroxyl and $4^{\prime}$-methoxy substituent in ring $B$, but possesses the $4^{\prime}$-hydroxyl moiety. Interestingly, flavonoids possessing the 4'-O-methyl structure (e.g. acacetin) or more hydroxyl groups in ring B (e.g. luteolin) did not show the positive effect on ATP levels under the applied conditions. The other aglycones caused no significant effect or only mild decrease (fisetin and naringenin) of ATP levels during the 12-h incubation period (Fig. 2a).

Thereafter, in a new series of experiments MDCK cells were treated again with $10 \mu \mathrm{M}$ flavonoid concentration under the same conditions except that the incubation time in this case was $24 \mathrm{~h}$. While acacetin, apigenin and diosmetin did not cause remarkable changes in ATP levels, other aglycones - with the exception of luteolin - resulted in a significant decrease of ATP/protein ratio compared to that of the control (Fig. 2b). Baicalein, catechin, daidzein, flavone, galangin, genistein, hesperetin, morin, myricetin, naringenin and quercetin caused similar effect: about 25-30\% reduction of ATP levels was observed. Chrysin resulted in lower (16\%) while fisetin caused higher (46\%) decrease of ATP than the above listed flavonoid aglycones. These results are in accordance with the previous investigations discussed in the introductory section [5-9]. Interestingly, after 24-h treatment a significant elevation (about 20\%) of ATP levels was caused by luteolin. As it will be discussed also in the next part (Section 3.2.), the effect of DIOS is of short duration: in the case of 24-h treatment cellular ATP
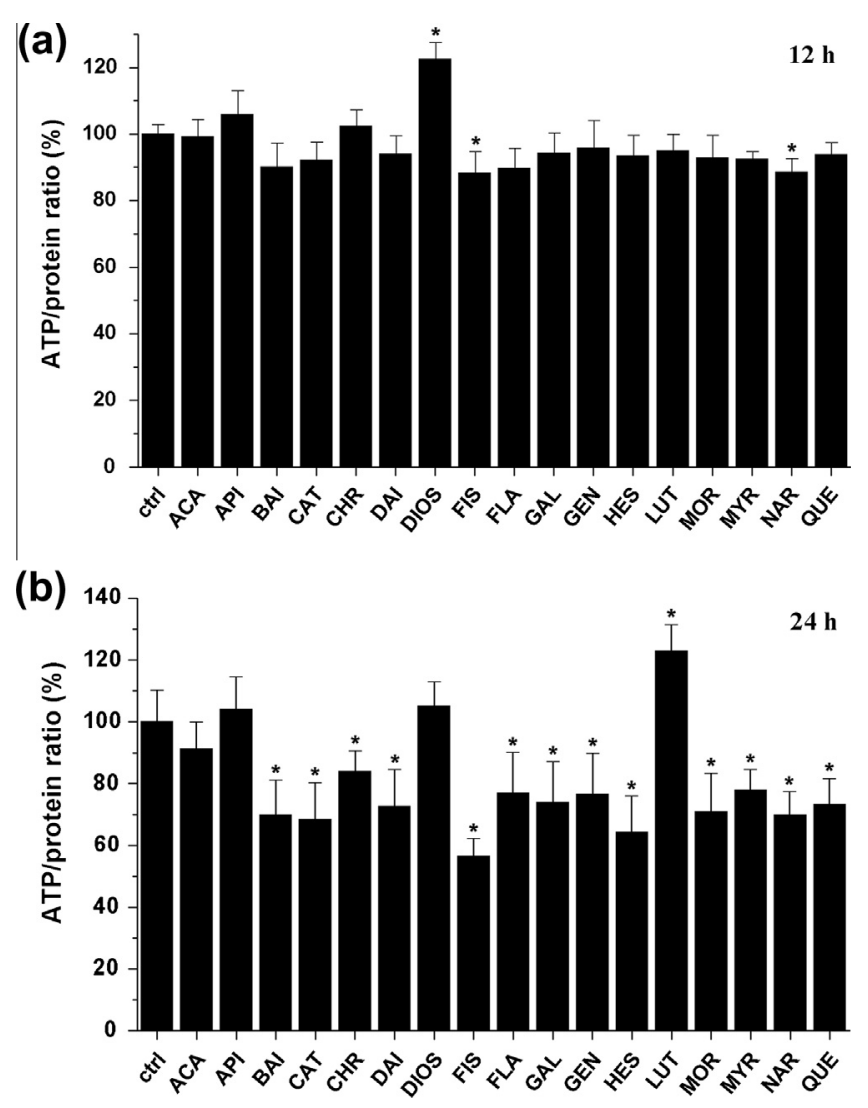

Fig. 2. ATP/total protein levels (in percentage, compared to the control) of MDCK cells after 12-h (above) and 24-h (below) treatment with $10 \mu \mathrm{M}$ flavonoid aglycones $\left({ }^{*} P<0.05\right)$. After 12 -h incubation only DIOS showed significant elevation of ATP levels furthermore fisetin and naringenin resulted in the significant decrease of ATP (a). Following 24-h treatment most of the flavonoids caused significant reduction of intracellular ATP while luteolin resulted in elevated ATP/protein ratio (b). [ctrl = control; ACA = acacetin; API = apigenin; $\mathrm{BAI}=$ baicalein; $\mathrm{CAT}$ = catechin; CHRY = chrysin $; \quad$ DAI = daidzein $; \quad$ DIOS = diosmetin $; \quad$ FIS = fisetin; $\quad$ FLA = flavone $\mathrm{GAL}=$ galngin; $\mathrm{GEN}=$ genistein; $\mathrm{HES}=$ hesperetin; $\mathrm{LUT}=$ luteolin; $\mathrm{MOR}=$ morin; MYR = myricetin; NAR = naringenin; $\mathrm{QUE}=$ quercetin $]$.

levels return to the control value. It is clearly seen in Fig. 1 that the only difference between DIOS and luteolin is the $4^{\prime}$-O-methyl moiety. Previous studies showed that DIOS can be metabolized to luteolin in liver cells both in vitro and in vivo, the demethylation is catalyzed by CYP1 isoenzymes [37,38]. On the other hand, recent in vivo investigations highlighted that luteolin is a substrate of the catechol-O-methyl transferase (COMT) enzyme and interestingly the most preferable reaction is its $4^{\prime}-O$-methylation which results in the formation of DIOS $[39,40]$. Since the activity of COMT enzyme in epithelial tubular kidney cells is high [41] and because luteolin did not increase ATP levels during 12-h treatment of MDCK kidney cells we hypothesize that the delayed (compared to DIOS) effect of luteolin on the ATP system is caused by the formation of its metabolic product DIOS.

\subsection{Effects of flavone diosmetin on ATP levels and viability of MDCK cells}

Based on the above described observations our attention was focused on flavone DIOS. To investigate the concentration and time dependence of the observed impact cells were treated with 5, 10 and $15 \mu \mathrm{M}$ DIOS concentrations and ATP levels were determined after 6, 12 and 24-h incubations (Fig. 3). After 6-h treatment, a dose dependent elevation of intracellular ATP/protein ratios was observed: Even $5 \mu \mathrm{M}$ DIOS led to $16 \%$ increase and higher 

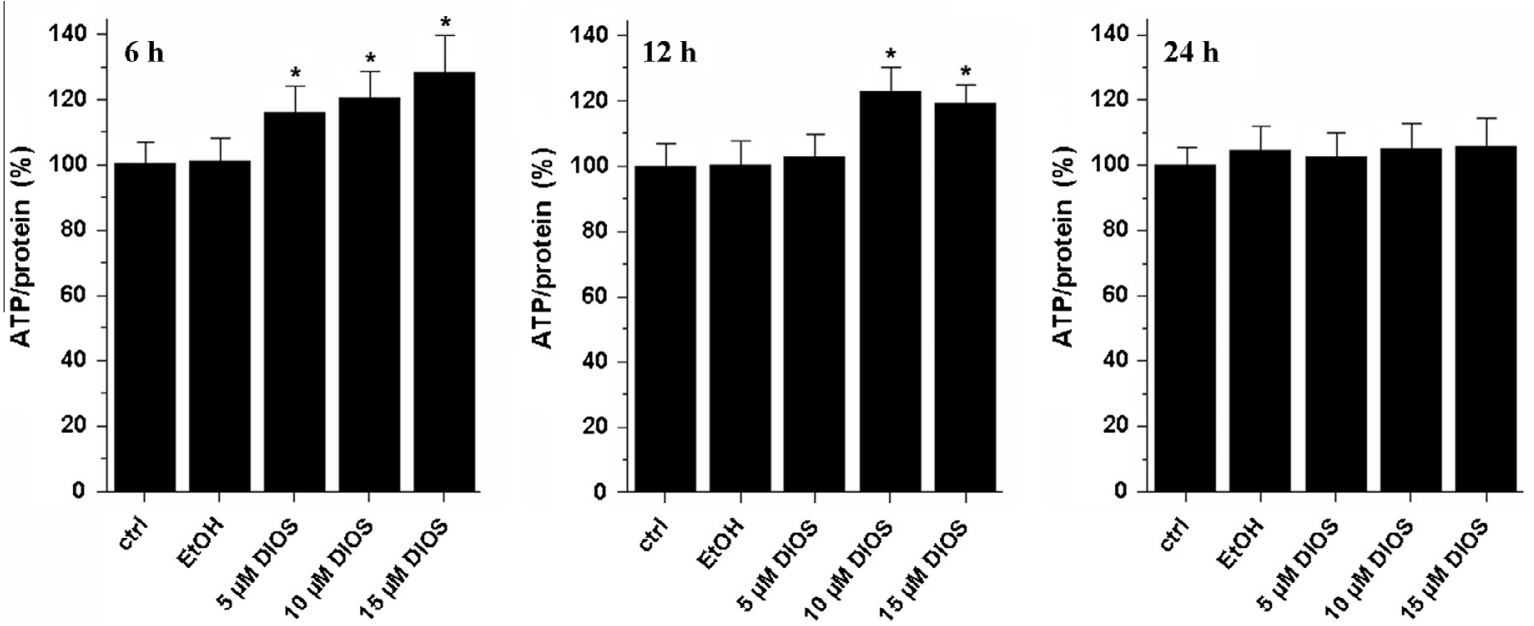

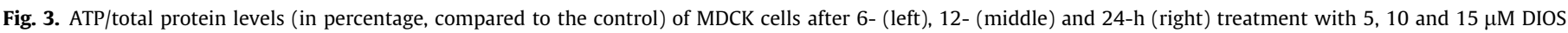

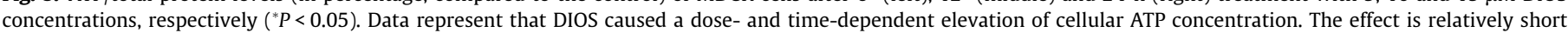
(6-12 h): after 24-h the ATP levels returns to the control value. [ctrl = control; EtOH = ethanol; DIOS= diosmetin].

concentrations (10 and $15 \mu \mathrm{M}$ ) enhanced this phenomenon (20\% and $28 \%$, respectively). Following 12 -h incubation $5 \mu \mathrm{M}$ DIOS did not inflict significant changes compared to the control. On the other hand, $23 \%$ increase was obtained at $10 \mu \mathrm{M}$ DIOS concentration. $15 \mu \mathrm{M}$ DIOS did not cause further elevation of ATP levels (compared to that of $10 \mu \mathrm{M}$ concentration). After 24-h treatment ATP levels were fallen down to the values obtained for controls for all three DIOS concentrations. Total protein contents and the results of calcein viability assay did not show significant differences compared to values of the controls at the applied conditions (data not shown), indicating that the number of living MDCK cells did not change during DIOS treatment. Thus, our results highlighted that under these circumstances DIOS was able to significantly elevate intracellular ATP levels, in a dose- and timedependent manner; furthermore, no viability loss of MDCK cells was observed during these experiments.

\subsection{Effects of diosmetin on ATP levels and ATP/ADP ratios of $M D C K$ cells (HPLC)}

After quantification of intracellular ATP levels with luciferinluciferase reaction (using detergent ATP extraction method), in order to verify our results and to determine the ATP/ADP ratios, confirmatory measurements were performed with HPLC using perchloric acid for the extraction. MDCK cells were treated with $10 \mu \mathrm{M}$ DIOS and after 12-h treatment ATP and ADP concentrations were quantified by HPLC-DAD system. Results are shown in Fig. 4 where determined ATP levels and ATP/ADP ratios of both the control and of the treated cells were expressed. These data are in good agreement with the results obtained from bioluminescent ATP determination: Significant increase of ATP concentration and considerable elevation of ATP/ADP ratio were observed.

\subsection{Effects of flavone diosmetin on the ATP levels and viability of HepG2 cells}

Thereafter to test the observed effect on a different cell line, additional measurements were performed using HepG2 liver cancer cells. Similarly to the MDCK cell line the elevation of ATP/protein ratios were detected after DIOS treatment. On the other hand it is important to note that a decrease of cell viability was also observed even in the case of lower DIOS concentrations based on total protein and calcein assays, suggesting a negative effect of DIOS on HepG2 cells. After 12-h incubation with 5-10 $\mu \mathrm{M}$ DIOS
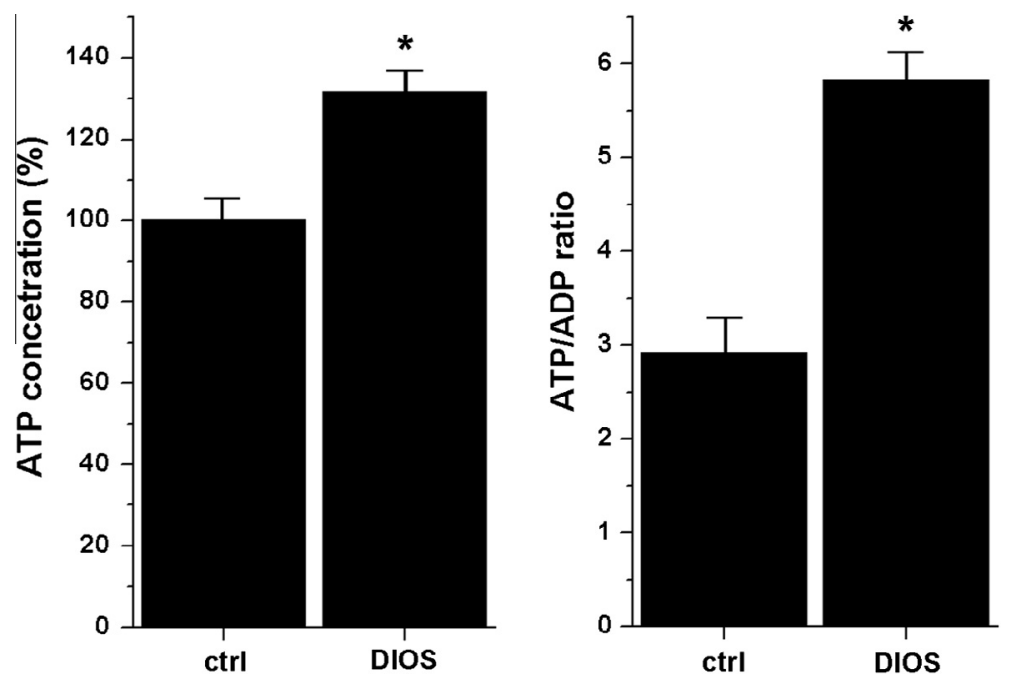

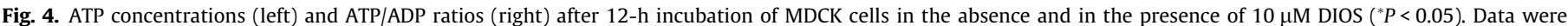

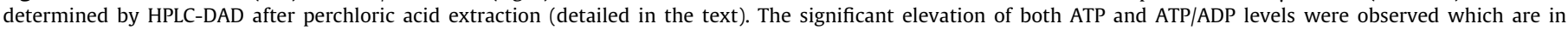
agreement with the results from the luciferin-luciferase ATP assay. [ctrl = control; DIOS= diosmetin]. 

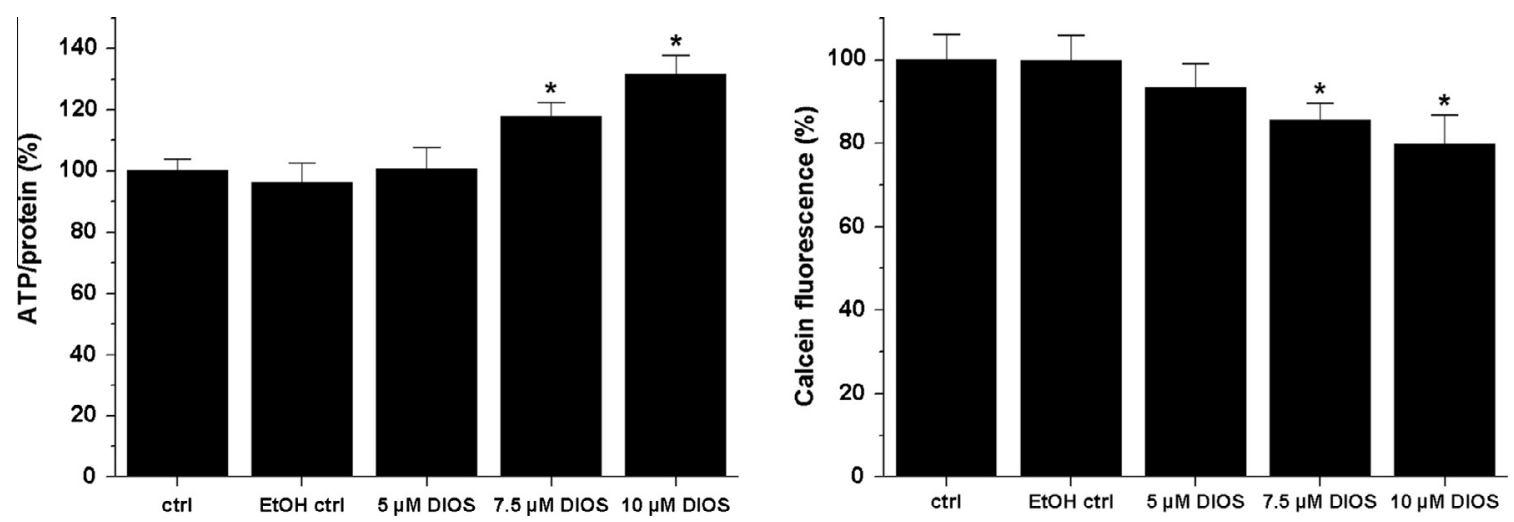

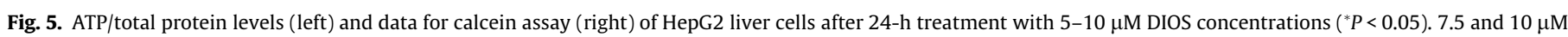

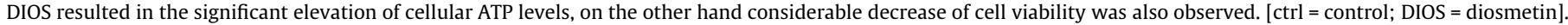

milder elevation of ATP/protein levels (8-11\%, respectively) was observed. On the other hand, 7.5 and $10 \mu \mathrm{M}$ DIOS caused a significant increase of ATP/protein ratios (18\% and $32 \%$, respectively) while considerable decrease of cell viability was also detected (15 and 20\%, respectively) after 24-h treatment. Fig. 5 shows the determined values of ATP/protein ratio and calcein fluorescence after 24-h treatment with 5-10 $\mu \mathrm{M}$ DIOS. Total protein levels were in good correlation with calcein measurements (data not shown). There could be several explanations for the time difference of the DIOS-mediated effect between MDCK and HepG2 cells. Although the cell medium was the same, the uptake kinetics of DIOS to MDCK and HepG2 cells can be different and other tissue-derived differences can also play a role. Furthermore, it is possible that the metabolism of DIOS in HepG2 cells results in the delayed effect compared to MDCK cells. The decreased viability in HepG2 cells can be explained by the commonly occurring tumor selective toxicity of several flavonoids [42-44], since MDCK is an immortalized, whereas HepG2 is a tumor cell line. This idea is supported by the study of Androutsopoulos et al. [45] where tumor selective negative effect of DIOS on cell cycle progression and cell proliferation were reported on breast cancer cells.

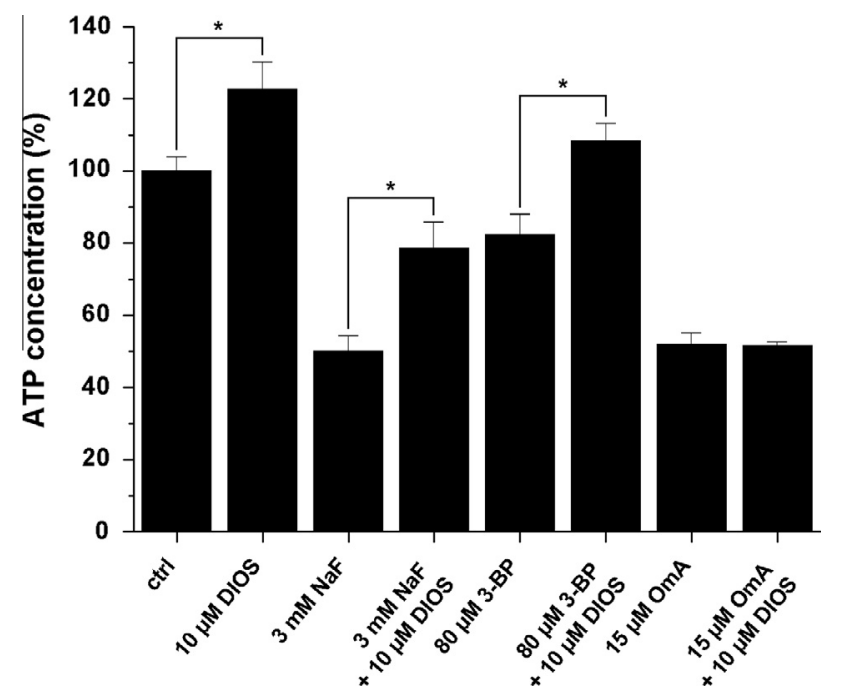

Fig. 6. ATP concentrations in MDCK cells after 12-h treatment with $10 \mu \mathrm{M}$ DIOS alone and co-treated with different enzyme inhibitors $\left({ }^{*} P<0.05\right)$. Applied sodium fluoride $(\mathrm{NaF}), 3$-bromopyruvate (3-BP) and olygomycin A (OmA) concentration caused a remarkable decrease of ATP levels. DIOS alone and in the presence of NaF or 3-BP was able to significantly elevate cellular ATP, on the other hand OmA completely abolished the positive effect of DIOS on ATP system. [ctrl = control; DIOS $=$ diosmetin] .

\subsection{Effects of enzyme inhibitors on diosmetin-induced ATP elevation (MDCK cells)}

In the next step of experiments different enzyme inhibitors were applied (using MDCK cells) in order to get more information on the observed DIOS-induced impact. Fig. 6 shows the measured ATP concentrations (applying bioluminescent ATP determination) after a 12-h combined treatment with DIOS and enzyme inhibitors. Our results show that negative effects of $\mathrm{NaF}(3 \mathrm{mM})$ and 3-BP $(80 \mu \mathrm{M})$ concentrations on ATP levels were considerably compensated by the simultaneous treatment with $10 \mu \mathrm{M}$ DIOS. These observations indicate that the inhibition of glycolysis or citric acid cycle cannot weaken or abolish the found impact. In contrast, complete inhibition of DIOS-induced increase of ATP production was caused by oligomycin A $(15 \mu \mathrm{M})$ treatment (Fig. 6). These observations suggest that the positive effect of DIOS may be related directly or indirectly to the ATP synthase enzyme or to oxidative phosphorylation.

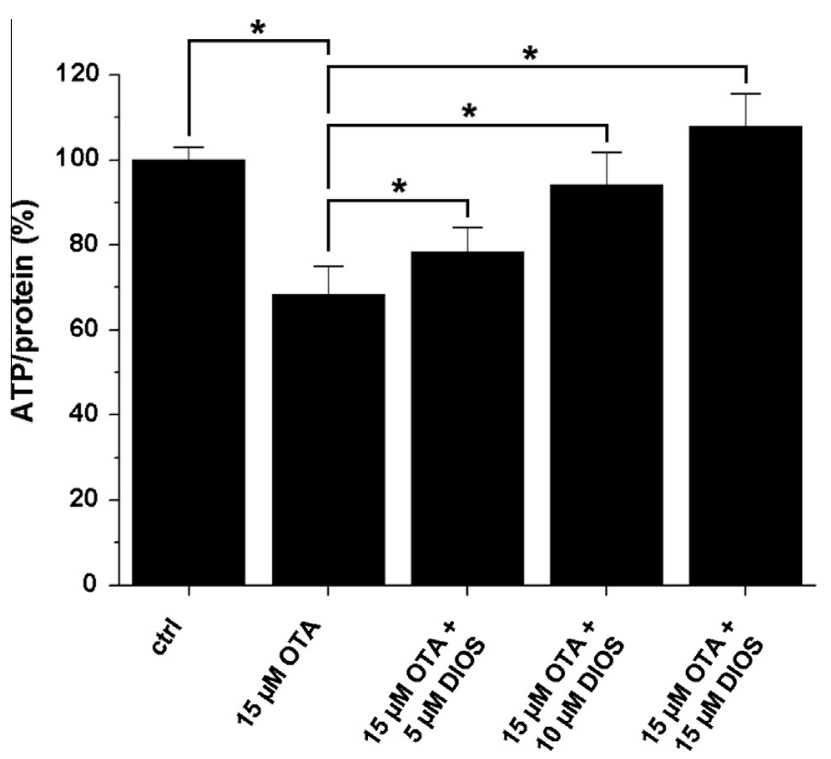

Fig. 7. ATP/total protein levels of MDCK cells after 24-h treatment with OTA alone and co-treated with $5-15 \mu \mathrm{M}$ DIOS (detailed in the text; ${ }^{*} P<0.05$ ). $15 \mu \mathrm{M}$ OTA resulted in considerable decrease (more than 30\%) of cellular ATP levels. Cotreatment with DIOS caused a dose-dependent, significant elevation of ATP/ADP ratio, $15 \mu \mathrm{M}$ DIOS abolished completely the OTA-mediated ATP loss. [ctrl = control; OTA = ochratoxin A; DIOS = diosmetin]. 
3.6. Effects of diosmetin on mitochondrial oxygen consumption (isolated rat liver mitochondria)

To test the potential involvement of ATP synthase in the action of DIOS, its effect on rat liver mitochondria was also examined. Surprisingly, the oxygen consumption of isolated mitochondria did not show any increase after treatment with 5-15 $\mu \mathrm{M}$ DIOS. Only higher DIOS concentrations ( $20 \mu \mathrm{M}$ or above) resulted in mild uncoupling effect (data not shown), which is exactly the opposite of what we expected. Because lower concentrations of DIOS (5$15 \mu \mathrm{M}$ ) did not increase the oxygen consumption of the isolated mitochondria we hypothesize that DIOS does not act directly on the ATP synthase enzyme. The indirect mechanism of DIOS could be resulted from several actions e.g. the modification of the gene expression or the modulation of different proteins. Since recent studies highlighted that flavonoids have diverse and very complex biochemical effects on living cells and organisms [1-4,17-22] further extensive investigations are reasonable to conduct in order to decipher the exact mechanism of action of DIOS.

\subsection{Diosmetin versus ATP depleting effect of ochratoxin A}

Finally, the discovered positive impact of DIOS on the ATP system was tested in a model using a mycotoxin, ochratoxin A. The ATP depleting effect of OTA is not clearly understood yet but previous studies suggest its influence on the mitochondrial respiratory chain caused by the inhibition of mitochondrial phosphate transport and/or the direct effect on the electron transport chain (inhibition of succinate-supported electron activities) [27]. Based on our preliminary measurements on MDCK cells, low OTA concentrations $(5-15 \mu \mathrm{M})$ cause major changes on ATP levels at least after 24-h treatment (data not shown). On the other hand, DIOS seems to be effective under shorter incubation times (6-12 h). Therefore MDCK cells were treated with $15 \mu \mathrm{M}$ OTA (in $150 \mu \mathrm{L}$ medium/well) and after 12-h incubation the system was supplemented/cotreated with $0-15 \mu \mathrm{M}$ DIOS (in $50 \mu \mathrm{L}$ DMEM/well, without the replacement of the previous $150 \mu \mathrm{L}$ medium) and cells were incubated for further $12 \mathrm{~h}$. Fig. 7 represents that under the applied conditions $15 \mu \mathrm{M}$ OTA resulted in about 30\% decrease of total protein-related ATP levels after the 24 -h incubation period. Furthermore, all applied DIOS concentrations lead to significant elevation of ATP/protein values of OTA-exposed cells. The impact is so effective that $15 \mu \mathrm{M}$ DIOS restore completely the intracellular ATP levels. Since ATP synthesis can be influenced by the oxidative status of the cells [46] antioxidant effect of DIOS was also tested applying the same conditions. $15 \mu \mathrm{M}$ OTA caused an about $60 \%$ increase of ROS production (Fig. 8). DIOS alone resulted in a mild reduction of ROS/total protein levels in MDCK cells. In addition, DIOS caused significant but not dramatic decrease of ROS/protein ratios in co-treated (both OTA and DIOS) cells compared to the simply OTA-exposed cells. In vitro antioxidant effect of DIOS was reported in previous studies $[47,48]$. On the other hand, DIOS has much lower radical scavenging activity in cell cultures compared to other flavonoids (e.g. catechin, quercetin or luteolin) [48-50]. It is also supported by the study of Ramyaa and Padma [51] where OTA-induced oxidative stress was substantially alleviated by quercetin. However, none of the other tested flavonoids caused significant elevation of ATP levels in OTA-exposed cells (data not shown). These results suggest that the DIOS-induced effect on intracellular ATP concentrations is independent from or depends only slightly on the antioxidant property of the flavonoid. It is also important to note, that based on the previous studies the ATPdepleting effect of OTA is resulted from the inhibition of mitochondrial respiratory chain but its toxic effect is not related directly to the ATP synthase enzyme $[27,52-54]$. On the other hand, DIOS abolished the negative effect of OTA on ATP system however, the inhibition of ATP synthase by olygomicin cannot be alleviated by DIOS. These observations also suggest the important role of ATP synthase in the action of DIOS but as we previously discussed the connection is probably indirect.

\subsection{Conclusions}

In our study the effects of flavonoids on cellular ATP levels were examined. We described for the first time the unexpected effect of DIOS on ATP levels. Our major conclusions are the following: DIOS
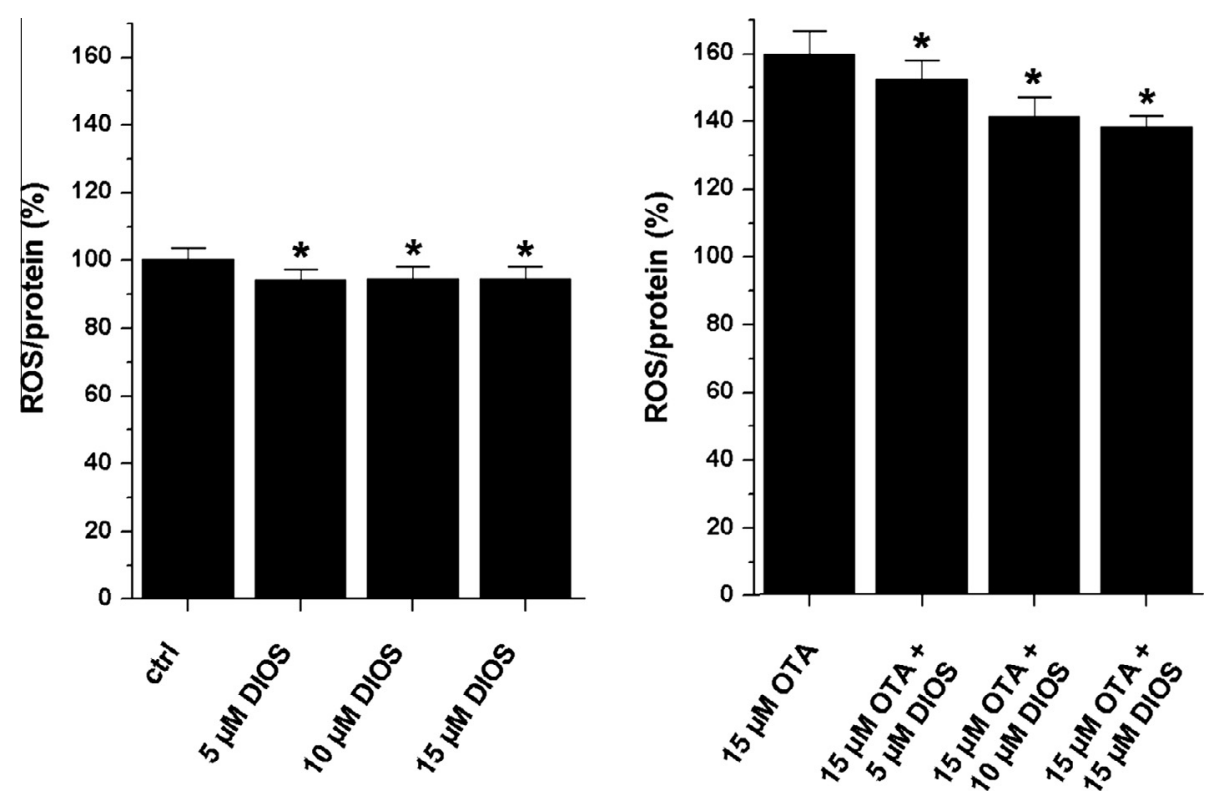

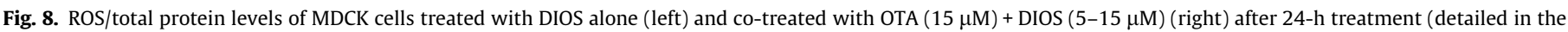

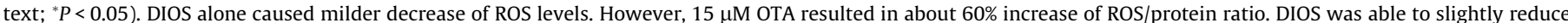

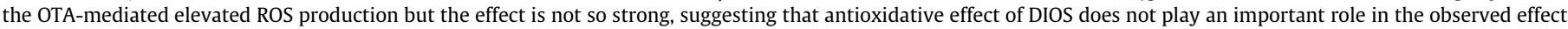
on ATP system. [ctrl = control; OTA = ochratoxin A; DIOS = diosmetin]. 
increases intracellular ATP levels both in MDCK and HepG2 cells. Inhibition of glycolysis or citric acid cycle does not suppress the observed effect. DIOS-induced elevation of ATP levels is completely abolished by the inhibition of ATP synthase with oligomycin A. DIOS is able to reverse the ATP-depleting effect of the mycotoxin, ochratoxin A. Most probably the DIOS-induced impact on ATP system does not originate from to the antioxidant property of DIOS. Based on our findings the above described special impacts of DIOS may be suitable to positively influence ATP-depletion caused by some metabolic poisons.

\section{Acknowledgements}

Our study was supported by PTE ÁOK-KA-2013/15 and PTE ÁOK-KA-2013/31. Financial support of the Developing Competitiveness of Universities in the South Transdanubian Region project (SROP-4.2.1.B-10/2/KONV-2010-0002) is highly appreciated.

\section{References}

[1] B.H. Havsteen, The biochemistry and medical significance of the flavonoids, Pharmacol. Therapeut. 96 (2002) 67-202.

[2] R.J. Korthui, D.C. Gute, Anti-inflammatory actions of a micronized, purified flavonoid fraction in ischemia/reperfusion, Adv. Exp. Med. Biol. 505 (2002) $181-190$.

[3] F. Dajas, Life or death: neuroprotective and anticancer effects of quercetin, J. Ethnopharmacol. 143 (2012) 383-396.

[4] V.H. Tran, D. Marks, R.K. Duke, M. Bebawy, C.C. Duke, B.D. Roufogalis, Modulation of P-glycoprotein-mediated anticancer drug accumulation, cytotoxicity, and ATPase activity by flavonoid interactions, Nutr. Cancer 63 (2011) 435-443.

[5] N. Chinnam, P.K. Dadi, S.A. Sabri, M. Ahmad, M.A. Kabir, Z. Ahmad, Dietary bioflavonoids inhibit Escherichia coli ATP synthase in a differential manner, Int. J. Biol. Macromol. 46 (2010) 478-486.

[6] B. Li, S.B. Vik, Y. Tu, Theaflavins inhibit the ATP synthase and the respiratory chain without increasing superoxide production, J. Nutr. Biochem. 23 (2012) 953-960.

[7] S. Kanno, A. Tomizawa, T. Ohtake, K. Koiwai, M. Ujibe, M. Ishikawa, Naringenininduced apoptosis via activation of NF- $\kappa \mathrm{B}$ and necrosis involving the loss of ATP in human promyeloleukemia HL-60 cells, Toxicol. Lett. 166 (2006) 131139.

[8] L. Mathiesen, K.E. Malterud, R.B. Sund, Uncoupling of respiration and inhibition of ATP synthesis in mitochondria by C-methylated flavonoids from Myrica gale L, Eur. J. Pharmac. Sci. 4 (1996) 373-379.

[9] D.J. Dorta, A.A. Pigoso, F.E. Mingatto, T. Rodrigues, I.M.R. Prado, A.F.C. Helena, S.A. Uyemura, A.C. Santos, C. Curti, The interaction of flavonoids with mitochondria: effects on energetic processes, Chem. Biol. Interact 152 (2005) 67-78.

[10] E. Tripoli, M. La Guardia, S. Giammanco, D. Di Majo, M. Giammanco, Citrus flavonoids: molecular structure, biological activity and nutritional properties: a review, Food Chem. 104 (2007) 466-479.

[11] S. Roowi, A. Crozier, Flavonoids in tropical citrus species, J. Agric. Food Chem. 59 (2011) 12217-12225.

[12] G. Gattuso, C. Caristi, C. Gargiulli, E. Bellocco, G. Toscano, U. Leuzzi, Flavonoid glycosides in bergamot juice (Citrus bergamia Risso), J. Agric. Food Chem. 54 (2006) 3929-3935.

[13] J. Meirinhos, B.M. Silva, P. Valentão, R.M. Seabra, J.A. Pereira, A. Dias, P.B. Andrade, F. Ferreres, Analysis and quantification of flavonoidic compounds from Portuguese olive (Olea europaea L.) leaf cultivars, Nat. Prod. Res. 19 (2005) 189-195.

[14] O. Benavente-García, J. Castillo, J. Lorente, A. Ortuno, J.A. Del Rio, Antioxidant activity of phenolics extracted from Olea europaea L. leaves, Food Chem. 68 (2000) 457-462.

[15] C. Amato, Advantage of a micronized flavonoidic fraction (Daflon $500 \mathrm{mg}$ ) in comparison with a nonmicronized diosmin, Angiology 45 (1994) 531-536.

[16] V.S. Savel'ev, A.V. Pokrovskiĭ, S.V. Sapelkin, I.V. Bogachev, I.L. Bogdanets, IA. Zolotukhin, Micronized diosmin (Detralex) for vein-related trophic ulcers: European experience, Angiol. Sosud. Khir. 12 (2006) 53-60.

[17] T. Tanaka, H. Makita, K. Kawabata, H. Mori, M. Kakumoto, K. Satoh, A. Hara, T. Sumida, T. Tanaka, H. Ogawa, Chemoprevention of azoxymethane-induced rat colon carcinogenesis by the naturally occurring flavonoids, diosmin and hesperidin, Carcinogenesis 18 (1997) 957-965.

[18] N. Alvarez, V. Vicente, C. Martínez, Synergistic effect of diosmin and interferon-alpha on metastatic pulmonary melanoma, Cancer Biother. Radiopharm. 24 (2009) 347-352.

[19] L. Pari, S. Srinivasan, Antihyperglycemic effect of diosmin on hepatic key enzymes of carbohydrate metabolism in streptozotocin-nicotinamide-induced diabetic rats, Biomed. Pharmacother. 64 (2010) 477-481.
[20] T. Silambarasan, B. Raja, Diosmin, a bioflavonoid reverses alterations in blood pressure, nitric oxide, lipid peroxides and antioxidant status in DOCA-salt induced hypertensive rats, Eur. J. Pharmacol. 679 (2012) 81-89.

[21] M.U. Rehman, M. Tahir, A. Quaiyoom Khan, R. Khan, A. Lateef, O.O. Hamiza, F. Ali, S. Sultana, Diosmin protects against trichloroethylene-induced renal injury in Wistar rats: plausible role of p53, Bax and caspases, Br. J. Nutr. 110 (2013) 699-710.

[22] M. Tahir, M.U. Rehman, A. Lateef, R. Khan, A.Q. Khan, W. Qamar, F. Ali, O O'Hamiza, S. Sultana, Diosmin protects against ethanol-induced hepatic injury via alleviation of inflammation and regulation of TNF- $\alpha$ and NF- $\kappa$ B activation, Alcohol 47 (2013) 131-139.

[23] D. Cova, L. De Angelis, F. Giavarini, G. Palladini, R. Perego, Pharmacokinetics and metabolism of oral diosmin in healthy volunteers, Int. J. Clin. Pharmacol. Ther. Toxicol. 30 (1992) 29-33.

[24] M.A. Campanero, M. Escolar, G. Perez, E. Garcia-Quetglas, B. Sadaba, J.R. Azanza, Simultaneous determination of diosmin and diosmetin in human plasma by ion trap liquid chromatography-atmospheric pressure chemical ionization tandem mass spectrometry: application to a clinical pharmacokinetic study, J. Pharmac. Biomed. Anal. 51 (2010) 875-881.

[25] A.E. Pohland, S. Nesheim, L. Friedman, Ochratoxin A: a review, Pure Appl. Chem. 64 (1992) 1029-1046.

[26] S.C. Duarte, A. Pena, C.M. Lino, Ochratoxin A non-conventional exposure sources - a review, Microchem. J. 93 (2009) 115-120.

[27] D. Ringot, A. Chango, Y.J. Schneider, Y. Larondelle, Toxicokinetics and toxicodynamics of ochratoxin A, an update, Chem. Biol. Interact. 159 (2006) $18-46$.

[28] M. Poór, Y. Li, S. Kunsági-Máté, Z. Varga, A. Hunyadi, B. Dankó, F.R. Chang, Y.C. $\mathrm{Wu}, \quad$ T. Köszegi, Protoapigenone derivatives: albumin binding properties and effects on HepG2 cells, J. Photochem. Photobiol. B 124 (2013) 20-26.

[29] N. Kochanowski, F. Blanchard, R. Cacan, F. Chirat, E. Guedon, A. Marc, J.L. Goergen, Intracellular nucleotide and nucleotide sugar contents of cultured CHO cells determined by a fast, sensitive, and high-resolution ion-pair RPHPLC, Anal. Biochem. 348 (2006) 243-251.

[30] P.N. Lowe, R.N. Perham, Bromopyruvate as an active-site-directed inhibitor of the pyruvate dehydrogenase multienzyme complex from Escherichia coli, Biochemistry 23 (1984) 91-97.

[31] M.C. Shoshan, 3-Bromopyruvate: targets and outcomes, J. Bioenergy Biomembr. 44 (2012) 7-15.

[32] W.C. Schneider, G.H. Hageboom, Further studies on the distribution of cytochrome c in rat liver homogenates, J. Biol. Chem. 183 (1950) 123-128.

[33] N.R. Sims, Rapid isolation on metabolically active mitochondria from rat brain and subregions using Percoll density gradient centrifugation, J. Neurochem. 55 (1990) 698-707.

[34] A. Elimadi, D. Morin, E. Albengres, A.M. Chauvet-Monges, V. Allain, A. Crevat, J.P. Tillement, Differential effects of zidovudine and zidovudine triphosphate on mitochondrial permeability transition and oxidative phosphorilation, Br. J. Pharmacol. 121 (1997) 1295-1300.

[35] W. Jakubowski, G. Bartosz, 2,7-dichlorofluorescin oxidation and reactive oxygen species: what does it measure?, Cell Biol Int. 24 (2000) 757-760.

[36] A. Grzelak, B. Rychlik, G. Bartosz, Light-dependent generation of reactive oxygen species in cell culture media, Free Radic. Biol. Med. 30 (2001) 14181425.

[37] V. Androutsopoulos, N. Wilsher, R.R. Arroo, G.A. Potter, Bioactivation of the phytoestrogen diosmetin by CYP1 cytochromes P450, Cancer Lett. 274 (2009) 54-60.

[38] V.P. Androutsopoulos, D.A. Spandidos, The flavonoids diosmetin and luteolin exert synergistic cytostatic effects in human hepatoma HepG2 cells via CYP1Acatalyzed metabolism, activation of JNK and ERK and P53/P21 up-regulation, J. Nutr. Biochem. 24 (2013) 496-504.

[39] Z. Chen, M. Chen, H. Pan, S. Sun, L. Li, S. Zeng, H. Jiang Role of catechol-Omethyltransferase in the disposition of luteolin in rats, Drug Metab. Dispos. 39 (2011) 667-674.

[40] Z. Chen, S. Kong, F. Song, L. Li, H. Jiang, Pharmacokinetic study of luteolin, apigenin, chrysoeriol and diosmetin after oral administration of Flos Chrysanthemi extract in rats, Fitoterapia 83 (2012) 1616-1622.

[41] P.T. Männistö, S. Kaakkola, Catechol-O-methyltransferase (COMT): biochemistry, molecular biology, pharmacology, and clinical efficacy of the new selective COMT inhibitors, Pharmacol. Rev. 51 (1999) 593-628.

[42] W.M. Alarif, A. Abdel-Lateff, A.M. Al-Abd, S.A. Basaif, F.A. Badria, M. Shams, S.E. Ayyad, Selective cytotoxic effects on human breast carcinoma of new methoxylated flavonoids from Euryops arabicus grown in Saudi Arabia, Eur. J. Med. Chem. 66 (2013) 204-210.

[43] F. Hakimuddin, G. Paliyath, K. Meckling, Selective cytotoxicity of a red grape wine flavonoid fraction against MCF-7 cells, Breast Cancer Res. Treat. 85 (2004) 65-79.

[44] A. Hunyadi, A. Martins, B. Danko, F.R. Chang, Y.C. Wu, Protoflavones: a class of unusual flavonoids as promising novel anticancer agents, Phytochem. Rev. (2013), http://dx.doi.org/10.1007/s11101-013-9188-2.

[45] V.P. Androutsopoulos, S. Mahale, R.R. Arroo, G. Potter, Anticancer effects of the flavonoid diosmetin on cell cycle progression and proliferation of MDA-MB 468 breast cancer cells due to CYP1 activation, Oncol. Rep. 21 (2009) 15251528.

[46] Z.T. Schafer, A.R. Grassian, L. Song, Z. Jiang, Z. Gerhart-Hines, H.Y. Irie, S. Gao, P. Puigserver, J.S. Brugge, Antioxidant and oncogene rescue of metabolic defects caused by loss of matrix attachment, Nature 461 (2009) 109-113. 
[47] P. Villa, D. Cova, L. De Francesco, A. Guaitani, G. Palladini, R. Perego, Protective effect of diosmetin on in vitro cell membrane damage and oxidative stress in cultured rat hepatocytes, Toxicology 73 (1992) 179-189.

[48] I. Morel, G. Lescoat, P. Cogrel, O. Sergent, N. Pasdeloup, P. Brissot, P. Cillard, J. Cillard, Antioxidant and iron-chelating activities of the flavonoids catechin, quercetin and diosmetin on iron-loaded rat hepatocyte cultures, Biochem. Pharmacol. 45 (1993) 13-19.

[49] S.R. Husain, J. Cillard, P. Cillard, Hydroxyl radical scavenging activity of flavonoids, Phytochemistry 26 (1987) 2489-2491.

[50] A. Seyoum, K. Asres, F.K. El-Fiky, Structure-radical scavenging activity relationships of flavonoids, Phytochemistry 67 (2006) 2058-2070.
[51] P. Ramyaa, V.V. Padma, Ochratoxin-induced toxicity, oxidative stress and apoptosis ameliorated by quercetin - modulation by Nrf2, Food Chem. Toxicol. 62 (2013) 205-216

[52] M.D. Aleo, R.D. Wyat, R.G. Schnellmann, Mitochondrial dysfunction is an early event in ochratoxin A but not oosporein toxicity to rat renal proximal tubules, Toxicol. Appl. Pharmacol. 107 (1991) 73-80.

[53] H. Meisner, S. Chan, Ochratoxin A, an inhibitor of mitochondrial transport systems, Biochemistry 13 (1974) 2795-2800.

[54] Y.H. Wei, T.N. Lu, R.D. Wei, Effect of ochratoxin A on rat liver mitochondrial respiration and oxidative phosphorilation, Toxicology 36 (1985) 119-123. 